A method for rapid production of subject specific finite element meshes for electrical impedance tomography of the human head

This article has been downloaded from IOPscience. Please scroll down to see the full text article.

2012 Physiol. Meas. 33801

(http://iopscience.iop.org/0967-3334/33/5/801)

View the table of contents for this issue, or go to the journal homepage for more

Download details:

IP Address: 128.40.160.155

The article was downloaded on 31/07/2012 at 15:23

Please note that terms and conditions apply. 


\title{
A method for rapid production of subject specific finite element meshes for electrical impedance tomography of the human head
}

\author{
Martin Vonach $^{1}$, Ben Marson ${ }^{1}$, Mark Yun ${ }^{1}$, Jorge Cardoso ${ }^{2}$, \\ Marc Modat $^{2}$, Sebastien Ourselin ${ }^{2}$ and David Holder ${ }^{1,3}$ \\ ${ }^{1}$ Department of Medical Physics, University College London, London, UK \\ ${ }^{2}$ Centre for Medical Image Computing (CMIC), University College London, London, UK \\ E-mail: d.holder@ucl.ac.uk
}

Received 9 December 2011, accepted for publication 3 April 2012

Published 24 April 2012

Online at stacks.iop.org/PM/33/801

\begin{abstract}
Finite element (FE) methods are widely used in electrical impedance tomography (EIT) to enable rapid image reconstruction of different tissues based on their electrical conductivity. For EIT of brain function, anatomicallyaccurate (head-shaped) FE meshes have been shown to improve the quality of the reconstructed images. Unfortunately, given the lack of a computational protocol to generate patient-specific meshes suitable for EIT, production of such meshes is currently ad hoc and therefore very time consuming. Here we describe a robust protocol for rapid generation of patient-specific FE meshes from MRI or CT scan data. Most of the mesh generation process is automated and uses freely available user-friendly software. Other necessary custom scripts are provided as supplementary online data and are fully documented. The patient scan data is segmented into four surfaces: brain, cerebrospinal fluid, skull and scalp. The segmented surfaces are then triangulated and used to generate a global mesh of tetrahedral elements. The resulting meshes exhibit high quality when tested with different criteria and were validated in computational simulations. The proposed protocol provides a rapid and practicable method for generation of patient-specific FE meshes of the human head that are suitable for EIT. This method could eventually be extended to other body regions and might confer benefits with other imaging techniques such as optical tomography or EEG inverse source imaging.
\end{abstract}

Keywords: mesh generation, electrical impedance tomography

(Some figures may appear in colour only in the online journal)

3 Author to whom any correspondence should be addressed. 


\section{Introduction}

\subsection{Background}

Finite element (FE) methods have been used to reconstruct numerically the underlying structure of an object, given its shape, by solving differential equations associated with different imaging modalities. For example, FE meshes have been employed in EEG source modelling (Baillet et al 2001, Drechsler et al 2009, Faugeras et al 2004, Lew et al 2009, Wolters et al 2007), optical tomography (Arridge 1999, Gibson et al 2005) and electrical impedance tomography (EIT) (Adler and Lionheart 2011, Metherall et al 1996, Soni et al 2004).

Our research group focuses on imaging of the brain using EIT. In EIT, images are usually produced from boundary voltages which are generated by the application of constant currents to other electrodes. The method employed in our group is to inject a constant current at a pair of electrodes, and record a voltage at a single different pair. Using a multiplexer, several hundred such measurements can be acquired in $1 \mathrm{~s}$, typically using multiple frequencies between 1 and $500 \mathrm{kHz}$. For image reconstruction in brain function with EIT, we employ a commonly used method based on the assumption of frequency independent quasi-static electromagnetic field propagation and a linear relation between changes in the subject and the boundary voltage (see (Holder 2005) for a review). A sensitivity matrix is generated, which describes the relation between conductivity changes in the model and changes in boundary voltages, using a forward solution of a generalized Laplacian equation for the FE method. Images are produced by calculating the inverse solution, in which ideally the recorded boundary voltages are multiplied by the inverse of the sensitivity matrix. There are typically only a few hundred measurements, compared to a few hundred thousand elements, which leads to an ill-posed inversion problem. Consequently a simple inversion of the sensitivity matrix is not possible and regularization is needed. Currently, we follow a truncated singular value decomposition strategy (Bagshaw et al 2003). This approach leads to reasonable images with a full width at half maximum of small test objects of about $10 \%$ of the image diameter in both computational and experimental simulations (Tidswell et al 2001).

Any numerical model should ideally contain an accurate representation of the brain and also the cerebrospinal fluid (CSF), the skull and scalp, given their differing electrical properties. The first FE meshes used in EIT brain imaging were single layered spheres (Tidswell et al 2001). This advanced to the use of multi-layered spheres representing different tissue layers (Liston et al 2004) and, most recently, to the use of a geometrically-accurate mesh (Tizzard et al 2005).

This first anatomically accurate mesh was constructed using commercial FEM generation software, I-DEAS, which required manual segmentation of MRI scan data (Tizzard et al 2005). By using an anatomically accurate mesh, the quality of the images obtained during phantom studies in a saline-filled head-shaped latex model was improved significantly. In particular, perturbation objects in the tank were better localized and less distorted than in the images obtained when using the layered sphere model (Bagshaw et al 2003). Unfortunately, each anatomically-accurate mesh required an input of about 20 man $\mathrm{h}$ from an experienced operator. Therefore, despite the associated increase in image quality, patient-specific meshes were not generated and a single prototype mesh was used across all patients. The anticipated use of such accurate meshes in neurological EIT could be to provide such a single prototype mesh for all subjects. It might also be used to provide subject specific meshes where CT or MRI were available - for example for monitoring following head injury or in patients being evaluated in a telemetry ward for epilepsy surgery. 
Several such studies (Bagshaw et al 2003, Kolehmainen et al 1997, Ramon et al 2006, Yerworth et al 2004) suggested that the use of more accurate anatomical meshes would improve the imaging results in EIT as well as in EEG source imaging. Therefore we are interested in creating patient-specific meshes for the purpose of image reconstruction in EIT. Several methods have been tested for generating accurate meshes for EIT imaging, including a self-refining multi-layered hemisphere able to refine areas with unacceptable errors (Towers et al 2000). The latter model presented benefits over the more simple models, though the self-refinement required multiple time consuming iterations to generate sensitivity matrices that were used to identify areas of high error. An alternative is to use textbook images to generate a prototype FE mesh with tissue layers (Bonovas et al 2001). This generated coarser meshes, but was faster to compute than the model proposed by Tizzard et al. More recently, the influence of including anisotropy information derived from diffusion tensor imaging into the FEM models for EIT (Abascal et al 2008, Abascal et al 2007) and EEG source imaging (Gullmar et al 2010, Hallez et al 2008, Rullmann et al 2009) has been investigated. The creation of subject specific FE meshes from patient scan data is a complex task performed in many areas of biomedical engineering (see (Sazonov and Nithiarasu 2012).

\subsection{Purpose}

The purpose of this work was to develop a rapid, robust and consistent method to generate patient-specific FE meshes from MRI or CT scan data. We describe a protocol which is accessible, rapid and could be undertaken with mainly freely available software suites with a graphical user interface. The method was specifically designed for EIT of human brain function using scalp electrodes. However, it is hoped that similar protocols could be used in EIT elsewhere in the body and that other imaging modalities, such as optical tomography or EEG inverse source imaging, could also benefit from the rapid generation of patient-specific FE meshes.

The method is presented with some examples of its use. Mesh quality was directly assessed by use of the 'scaled Jacobian', the 'condition number' and other standard criteria (for further details refer to the CUBIT website (Sandia Corporation 2012)). The accuracy of the resulting meshes was initially assessed by visual inspection, paying special attention to the thickness of each segmented layer. The resulting meshes were then successfully used for EIT simulations, producing similar images to those obtained with the manually-segmented mesh used in Tizzard et al (2005).

\section{Methods}

In this section, the protocol for the generation of patient-specific FE meshes is described. First, an overview and an explanation of the principles used in the protocol are provided. Secondly, a more technical description of the protocol is offered, and this is illustrated with seven MRI and five CT scans. Finally, the quality of the resulting meshes was analysed.

\subsection{Overview and explanation of the principles}

In this paper, we describe a protocol to generate FE meshes from patient scan data. The proposed protocol comprises four steps: segmentation, surface extraction, surface mesh processing and meshing (figure 1).

For EIT of brain function, the patient scan data needs to be segmented into four surfaces: brain, CSF, skull and scalp. Intensity differences across the layers depend highly on the imaging 


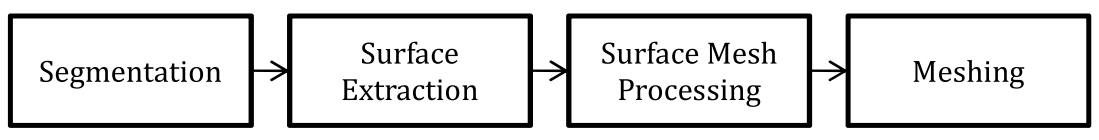

Figure 1. Steps in mesh creation.

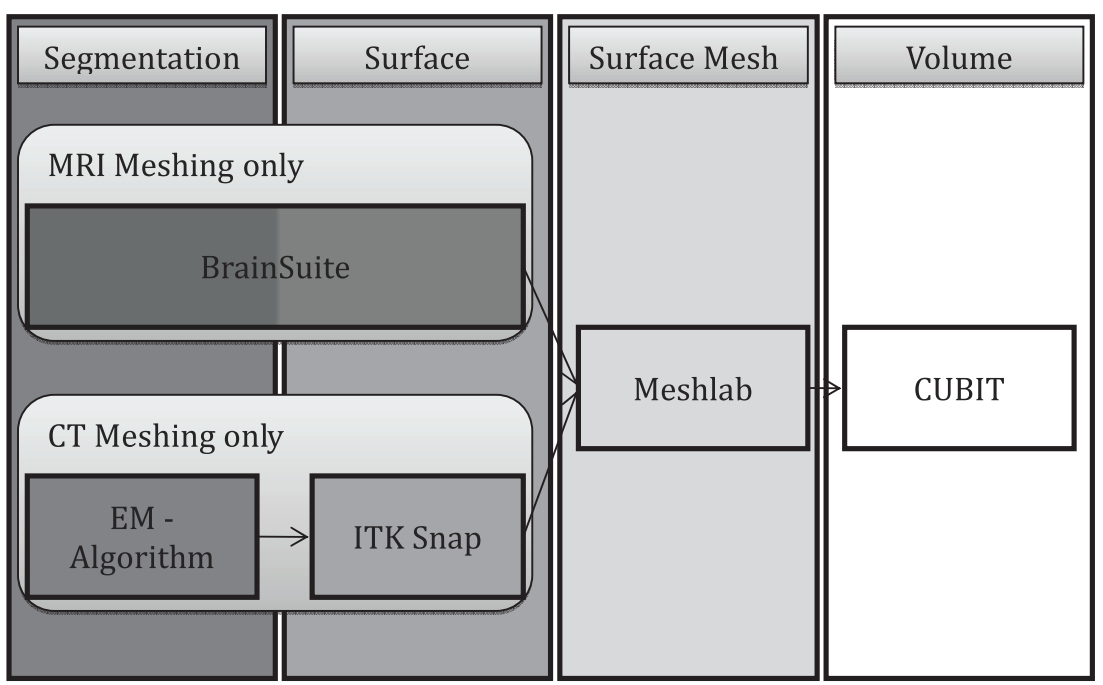

Figure 2. Software used in the four main steps of MRI and CT meshing.

modality (MRI or CT), as the contrast of different tissues varies. Therefore the segmentation step requires different algorithms for each imaging modality.

Once the four layers are segmented, their associated surfaces are extracted using a marching cubes algorithm (Lorensen and Cline 1987). This results in a triangulated surface mesh for each layer. These surface triangulations are then processed, in order to modify the number, size and shape of the elements contained in the final mesh. In the final step, a volumetric mesh of the whole head is generated, which comprises tetrahedral elements generated from the surface triangulations.

The software used for these four steps (see figure 2) is freely available and user-friendly. However, it was necessary to write some custom scripts in Matlab (Mathworks 2012) at some stages and to convert the file format across steps. Such scripts are available online (UCL 2012) and can also be requested from the corresponding author.

2.1.1. Segmentation and surface extraction. Prior to the segmentation, scan data was converted into the Analyse format which comprises a separated header and image files (see table 1 for an explanation of file formats encountered in the process). This conversion was performed using MRIcro, a freeware program intended for display of medical images (Rorden 2012).

Segmentation and surface extraction of MRI scan data. The open-source software BrainSuite (UCLA Laboratory of Neuro Imaging 2009) was used to segment the four different layers of the mesh. This software (chosen after careful consideration of available resources) is designed 
Table 1. File types used in meshing protocols.

\begin{tabular}{|c|c|c|}
\hline File name & Extension & Use \\
\hline ANALYSE & .hdr/.img & $\begin{array}{l}\text { Format developed by the MAYO clinic for describing MRI voxel } \\
\text { values (Wideman 2003). Data can be read in the } \\
\text { closely related Nifti format (Neuroimaging Informatics } \\
\text { Technology Initiative 2007). }\end{array}$ \\
\hline DUFF & .dfs & $\begin{array}{l}\text { BrainSuite file format describing location and connectivity of } \\
\text { triangles on a surface. }\end{array}$ \\
\hline Stereolithography & .stl & $\begin{array}{l}\text { File containing information defining surface triangles. Three vertices } \\
\text { with }(\mathrm{x}, \mathrm{y}, \mathrm{z}) \text { coordinates are given in an anticlockwise orientation } \\
\text { as well as a unit vector normal to the surface. (StereoLithography } \\
\text { Interface Specification, 3D Systems, Inc., October 1989) }\end{array}$ \\
\hline I-DEAS universal & .unv & $\begin{array}{l}\text { Output from the I-DEAS meshing software that contains information } \\
\text { defining elements (tetrahedrons). This information is stored } \\
\text { with predefined data identification reference numbers for } \\
\text { identification by other programs. }\end{array}$ \\
\hline MATLAB data & .mat & $\begin{array}{l}\text { The FEM is imported into MATLAB and saved in three matrices. } \\
\text { The first one contains the node coordinates, the second one is } \\
\text { the connectivity matrix and the last one contains a tissue } \\
\text { specific value for each element. }\end{array}$ \\
\hline
\end{tabular}

to identify different tissue types and surfaces in MRI data of the human head in a semiautomatic fashion, and requires little input from the user. It employs a combination of an edge-preserving and a Marr-Hildreth filter to isolate the brain followed by threshold detection to segment the scalp, outer and inner skull surfaces (Shattuck and Leahy 2002, Shattuck et al 2001). The output from this process was a '.dfs' file in which each layer surface is represented as triangular facets, in addition to metadata and patient information. The segmented surfaces were sometimes further processed (using BrainSuite) to eliminate any artefacts created during the segmentation process in order to obtain smooth layer surfaces. The resulting '.dfs' files were then converted to '.stl' files using a custom Matlab script. Both formats are similar, as each facet is described by a normal and the XYZ coordinates of each vertex.

Segmentation and surface extraction of CT scan data. While multiple pieces of open-source software can be used to perform accurate MRI segmentation, no freely-available software could be identified to perform the same task with the same level of precision for CT data. The main problem was the segmentation of the CSF layer, which is hardly visible in CT scans due to the low soft-tissue contrast of this imaging modality. Therefore, a custom hierarchical expectation maximization (EM) algorithm 'StartsegmentationAndRegistration' was developed that is able to segment the four layers from CT data. The segmentation step was based on an atlas that was created specifically for the purpose of CT segmentation. It contained the three-dimensional probability distribution for each tissue layer and was derived from several manual CT segmentations. This prior knowledge was registered to the patient CT using the multi-modal registration tool Nifty Reg (Modat 2012).

This segmentation algorithm was implemented in Matlab and $\mathrm{C}++$. The Statistical Parametric Mapping (SPM) package, a widely used program for analysis of medical images, is required to run the algorithm (Functional Imaging Laboratory 2012).

As the segmentation results were very detailed, additional processing was necessary to ensure that only the outer surface layers were extracted. For this purpose, custom written 
Matlab scripts were used (CreateSurfaces), in which binary and morphological operations were used.

The freely available software ITK-Snap (2011) was then used to create surface meshes based on the segmentation results. This software is mainly designed to allow manual and semi-automatic segmentation. The extracted surfaces were saved as .stl files.

2.1.2. Surface mesh processing. Surface mesh processing was performed using the freely available software (MeshLab 2012). MeshLab offers a range of sophisticated mesh processing tools with a graphical user interface.

The .stl files were imported into MeshLab, where they were processed and resampled using the so-called iso-parameterization algorithm. This algorithm calculated an almost-isometric surface triangulation, while preserving the main shape of the volume (Pietroni et al 2010). By choosing the resampling rate, the number of elements that the final mesh contained and the according element size were varied. Finally, the surfaces were again saved in the 'stl' format.

While the iso-parameterization filter could be applied to the MRI surfaces directly, further processing was needed for the surfaces extracted from CT scans. In particular, it was necessary to ensure that each layer surface described a single enclosed volume. The detailed surface also sometimes needed to be smoothed in order for the iso-parameterization algorithm to run successfully. MeshLab filters for smoothing, filling holes and deleting isolated volumes were used for this purpose.

2.1.3. Meshing. The processed surface meshes of the different layers were then loaded into CUBIT. This software is a toolkit for robust generation of 2D and 3D FE meshes developed by Sandia National Laboratories ( 2012). It generates quadrilateral and triangular surface paving, as well as hexagonal and tetrahedral meshing, and it also provides metrics of mesh quality. Using CUBIT, tetrahedral meshes from the surface triangulations were generated for each layer.

While the solid tetrahedral mesh for the brain layer was created using the inner brain surface only, the hollow outer layers needed to be meshed based using both outer and inner surface layers. This was achieved by subtraction of the two surfaces from each other. Then the volume defined by the different surfaces was meshed with tetrahedral elements using an advancing front algorithm. The four meshed volumes were exported as I-DEAS (.unv) files, and then were imported into Matlab by a custom Matlab script ('createFinalMesh'). Within Matlab, the four volume meshes were combined to form a unique head-shaped model with different properties for the four distinct tissue layers.

File types employed are summarized in table 1.

\subsection{Technical description of the segmentation and meshing procedure}

This section provides a more technical description of the different steps of the mesh generation protocol. Meshes for 12 test cases (seven MRI scans and five CT scans) were generated. The MRI datasets were obtained from the 'BrainWeb' publicly available database of simulated MRI scans (McConnell Brain Imaging Center 2006). CT scan datasets were obtained with permission from an EIT study at University College London Hospital.

2.2.1. Conversion and pre-processing. MRI or CT images were imported into MRIcro and rescaled in order to reduce computational demands. This software was also used to convert both MRI and CT data into the Analyse format used in subsequent steps. 


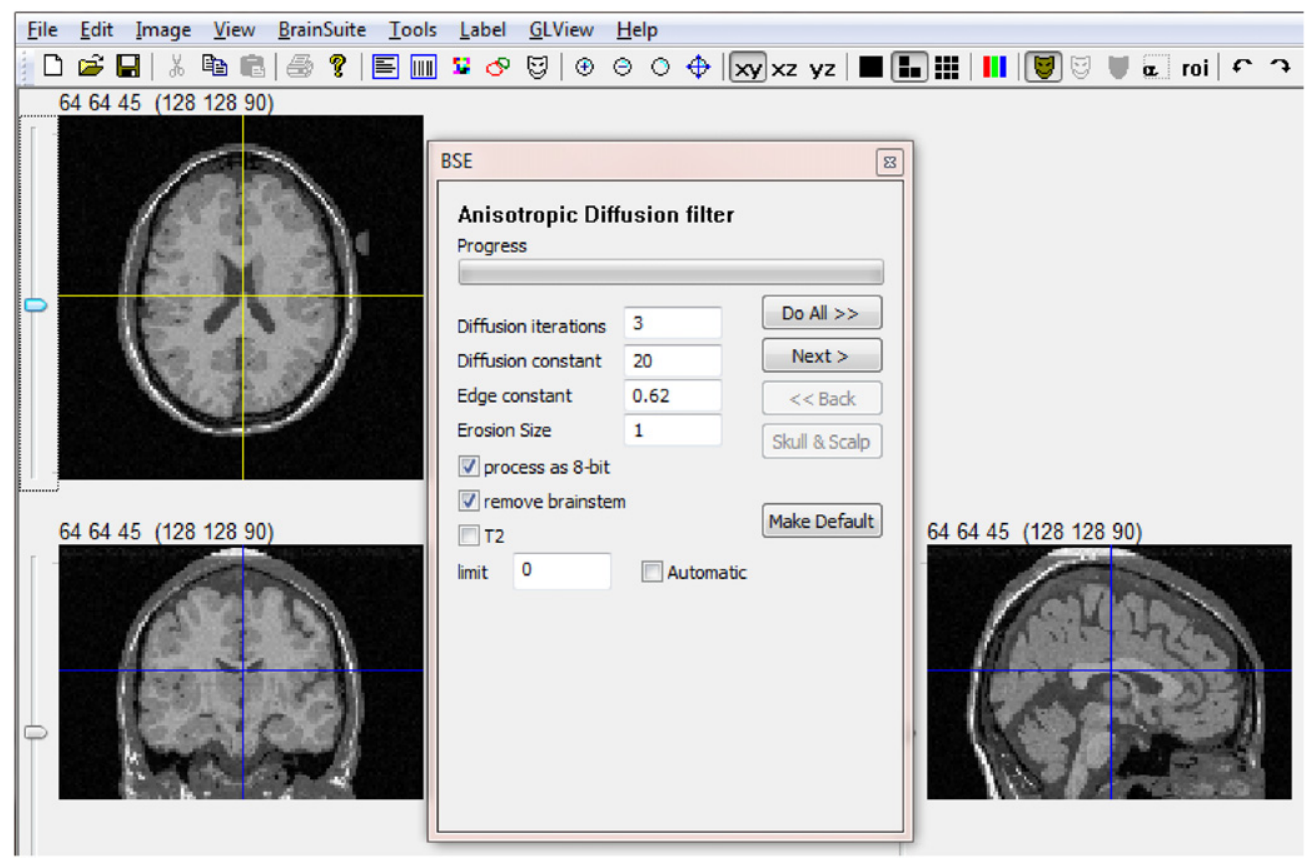

Figure 3. BrainSuite settings for skull stripping.

\section{Segmentation and surface extraction.}

MRI procedure. Analyse format MRI files were loaded into BrainSuite, and the four brain layers were segmented using the 'skull stripping (BSD)' tool. For the MRI test case, the brain layer was first segmented using values of three iterations, diffusion constant of 25 and edge constant of 0.62 (figure 3). The other layers were subsequently segmented using the 'Skull $\&$ Scalp' option with automatic computation of thresholds. The segmented surfaces were saved as '.dfs' files through the 'Surface View Properties' window that could be found by right-clicking on the opening three-dimensional view.

File format conversion of '.dfs' to 'stl' surface files. In the Matlab environment, '.dfs' files were read into memory using a custom Matlab function 'readdfs', which is based on a similar C++ function developed by David (Shattuck 2012), one of the authors of BrainSuite. The surfaces were converted and exported into '.stl' format using our custom written Matlab routine. This was repeated separately for each of the four surfaces.

CT procedure. Segmentation can be subdivided into the following three parts that was performed by running a single custom written Matlab script (StartsegmentationAndRegistration).

\section{(a) MRI to CT registration using Nifty Reg}

The freely available multi-modal registration software Nifty Reg (Modat 2012) was used to register the prior knowledge to the patient CT in three steps. First, an affine registration was estimated by using the Nifty Reg command 'reg_aladin'. Secondly, the transformation was 

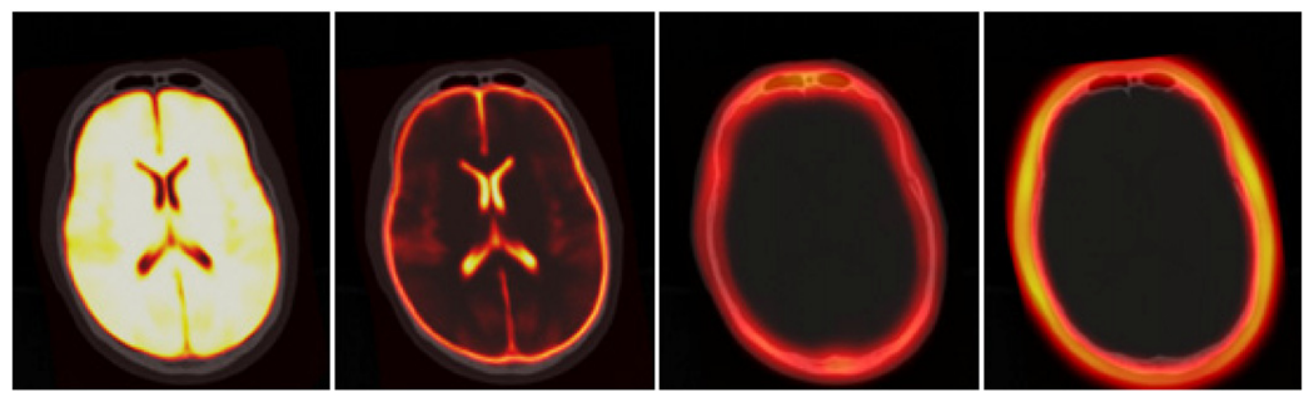

Figure 4. False colour overlay of registered atlas on patient CT; layers from left to right: brain, CSF, skull, scalp.

refined in a nonlinear approach using the command 'reg_f3d'. Finally, 'reg_resample' was used to apply the transformation to the four atlas files containing the prior knowledge.

Registration of the prior knowledge associated MRI atlas.nii to the patient data patientCT.nii was produced by entry of the following into the command line:

'reg_aladin -source atlas.nii -target patientCT.nii -aff aff.txt -ln 3-lp 2'

'reg_f3d -source atlas.nii -target patientCT.nii -aff aff.txt -cpp cpp.nii -ln 5-lp 3'

'reg_resample -source atlasBrain.nii -target patientCT.nii -aff aff.txt -cpp cpp.nii -result atlasBrainResult.nii'

where the -source and -target parameters specified which source image had to be registered to which target image. The estimated transformations were saved in the files given in parameters -aff and -cpp. - ln and -lp controlled the pyramidal approach iterations and -result specified the name of the registered image (figure 4).

(b) Expectation maximization (EM) segmentation

This code contains mex files that needed to be compiled on the system in order to run the segmentation. The segmentation algorithm incorporated prior relaxation (Cardoso et al 2011) and used contextual information to reduce noise using Markov random Fields (Van Leemput et al 1999). The segmentation code was run in Matlab, leading to reliable segmentation of the four tissue layers, including the ventricles as part of the CSF layer. The segmentation resulted in a probability volume for each layer.

(c) Segmentation processing

Using Matlab, the four probability images were transformed into binary segmentation masks by classifying each voxel according to the highest of the four probabilities, followed by creating a binary mask for each layer. This was accomplished using the custom Matlab script 'CreateSurfaces'. The outer tissue boundaries that separated the layers from each other were then be determined by fusion of these binary masks (figure 5).

Any gaps which occurred were filled by applying a morphological closing operation, which uses 2D structure elements to dilate the binary image first and to then apply erosion, with the effect that small holes are removed. The binary skull mask was then united with the brain mask and a CSF mask that was dilated by a $3 \times 3 \times 3$ matrix. The dilation ensured that there was a thin skull layer all around the CSF, which simplified the later meshing process, as a layer subtraction approach was used to mesh the volume.

In cases where the segmented CSF layer was too thin, the brain layer was eroded by a $2 \times 2 \times 2$ matrix, creating an artificial CSF layer when using the subtraction approach. 

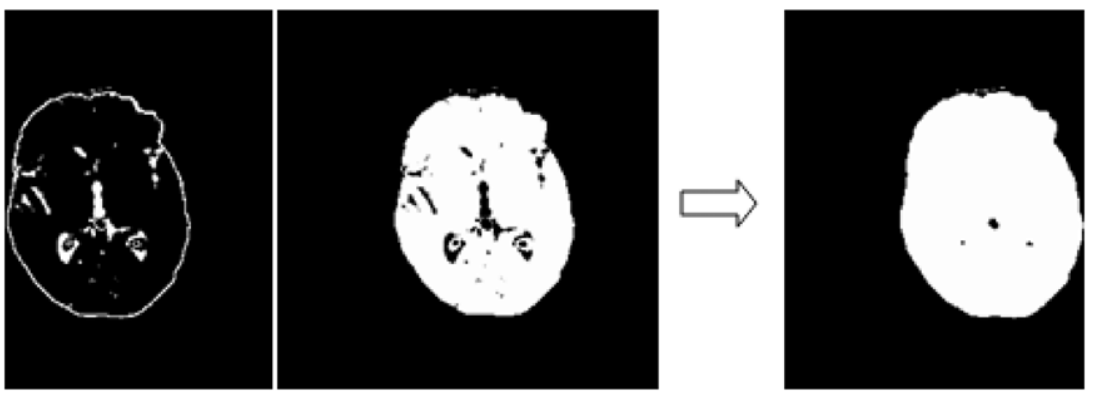

Figure 5. Union of binary segmentation masks; left: CSF; middle: brain; right: union.

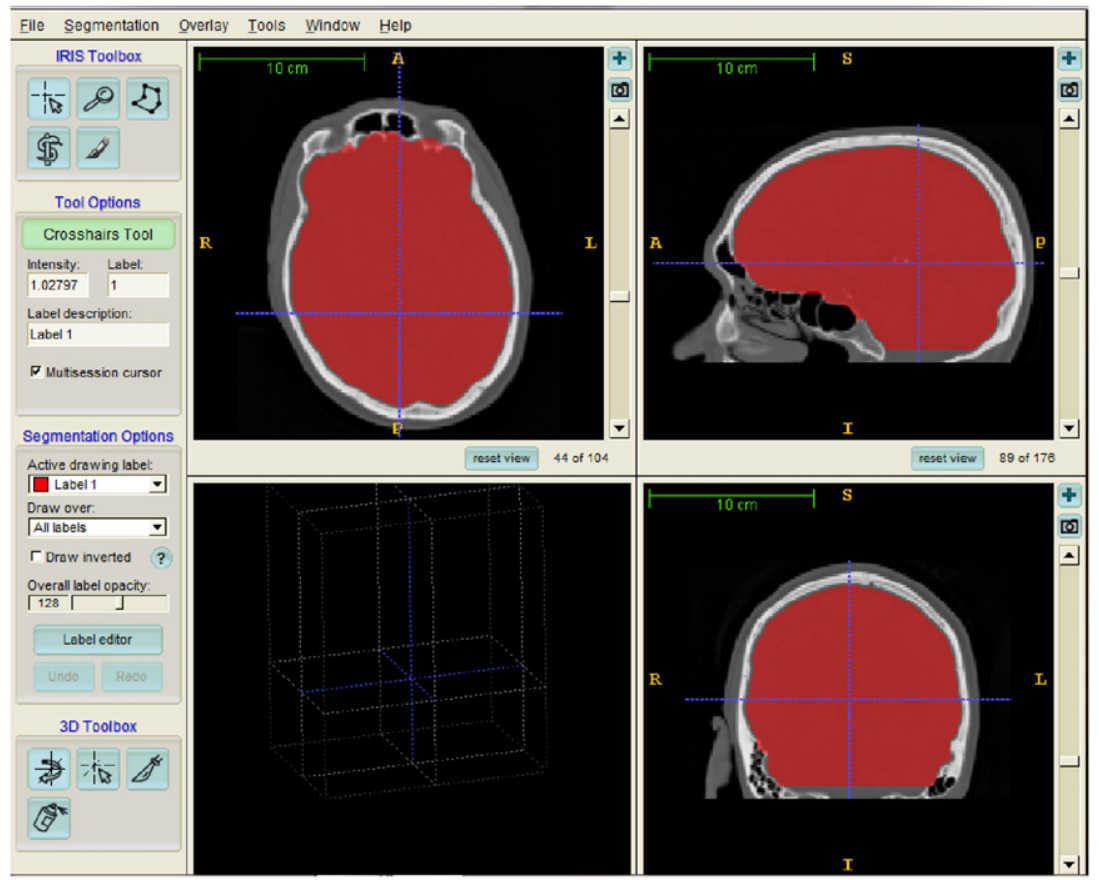

Figure 6. Binary segmentation mask visualized in ITK-Snap before export.

Finally, a surface mesh for each solid layer was created using ITK-Snap. The binary image was imported using ITK-Snap's 'import segmentation as image' function. The layer was then visualized as shown in figure 6 and could be visually inspected. Stl surface files were created by using the function 'save segmentation as mesh', which called a marching cube algorithm.

2.2.3. Surface mesh processing. The Marching Cubes algorithm produced very detailed surface meshes with a high number of small and irregular sized elements. Sometimes, typical staircase artefacts occurred. The surface meshes were therefore processed in order to be able to create meshes with reasonable quality. The open-source program MeshLab was used for this purpose. 


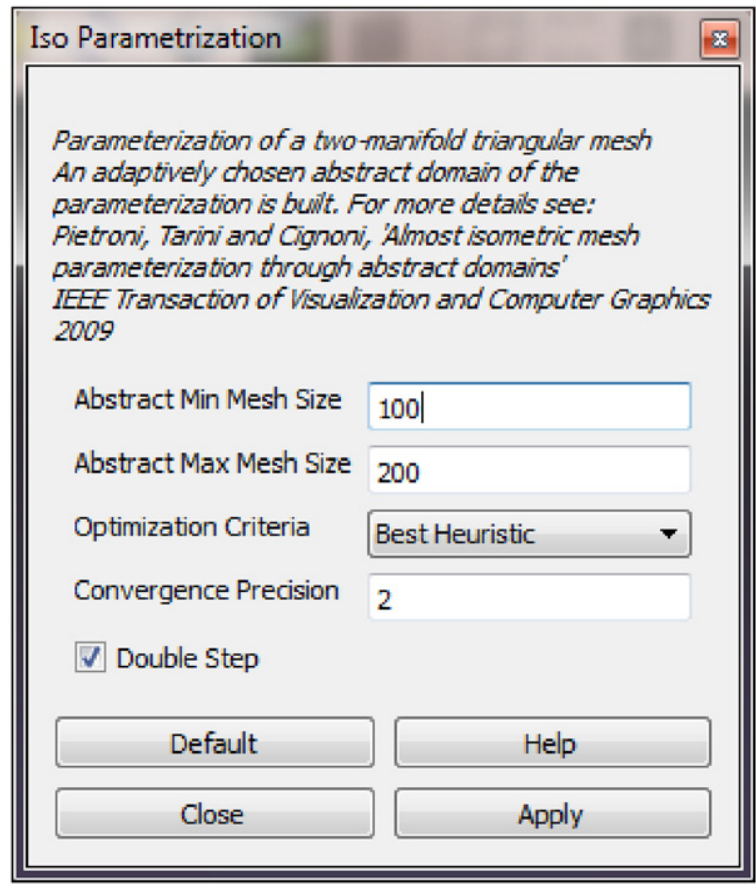

Figure 7. Iso-parameterization parameters.

Each layer surface was imported separately into MeshLab. For the MRI test case, BrainSuite implicitly smoothed and simplified the surfaces. Then, the iso-paramerization filter could be applied without any further processing. The standard parameters were used as indicated in figure 7.

In contrast, the surface meshes from the $\mathrm{CT}$ procedure were processed before running the iso-parameterization. In particular, the surface needed to be single-connected and watertight. By using the 'select small components' filter and deleting the selected set of vertices and faces, the mesh was reduced to a single component mesh if needed. Then, application of the 'Surface Reconstruction: Poisson' filter ensured that the model was watertight. However, the surface shape was sometimes affected if this filter was applied with standard parameters (figure 8). For the MRI test case, the shape of the surface was better preserved when the Octree depth parameter was changed to 7 .

Based on the abstract domain computed by the iso-parameterization filter, the volume was resampled using MeshLab's 'Iso-parameterization Remeshing' filter. The sample rate was chosen depending on the targeted element size. Higher sampling rates resulted in a smaller element size and an increased number of elements, compared to low sampling rates. A sampling rate value of 6 was used for the example meshes presented in this paper. Adaptive meshing could be achieved by choosing different sampling rates for different layers. The four resulting remeshed surfaces were saved as .stl files.

2.2.4. Meshing. The four surface files in .stl format were loaded into CUBIT with a feature angle of 0 , to ensure that each surface was imported as a single unbroken surface. The innermost layer was imported first, and then each sequential layer, so then the CSF, then skull, and lastly, scalp. 


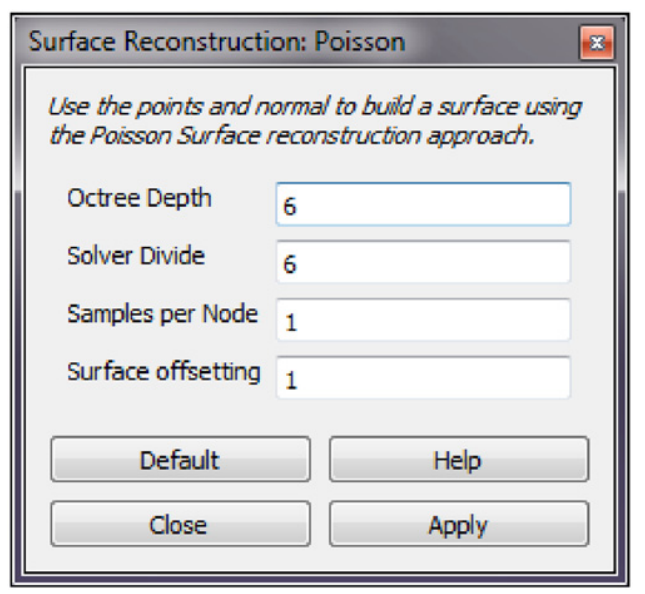

Figure 8. Poisson surface reconstruction parameters.

Individual tissue layers were produced by subtracting subsequent volumes. For this purpose the volumes of sequential surface meshes (scalp and skull, skull and CSF, CSF and brain) were then subtracted from one another using the volume Boolean function in the GUI to subtract the inner layer from their immediately lateral surface. This resulted in three hollow layer volumes with matching surface triangulations. The fourth volume consisted in the solid brain layer. The final layer mesh was then generated by typing

'mesh volume all from facets'

into the command line. CUBIT used the existing surface triangulations and extended the surface mesh into a tetrahedral volume mesh. Each layer was saved in a separate Universal I-DEAS (.unv) file using the export function in Cubit.

2.2.5. Matlab conversion. A custom conversion script written in Matlab (createFinalMesh) read in the four layer meshes from the previously exported unv files. The four meshes were united in one FE mesh, by combining the separate node lists and connectivity matrices, and created an element list with an entry representing the tissue type. This final mesh was then ready to be used in our bespoke EIT image reconstruction code.

\subsection{FEM evaluation}

2.3.1. Mesh size evaluation. The resulting meshes were validated in two main ways.

(1) By computing indices of mesh quality, such as the Jacobian, the scaled Jacobian, distortion and condition number. The condition number measures the distance of an element from the set of degenerate (non-convex or inverted) elements. Optimization of the condition number increases this distance and improves the shape quality of the elements. These metrics were calculated using inbuilt functions in CUBIT.

(2) By performing EIT simulations. A perturbation object was positioned at a known position in the computational domain. A forward simulation was run to obtain the boundary voltages associated with the conductivity field. These simulated voltages were used to reconstruct the conductivity field using the FEM mesh described and inversion of a sensitivity matrix with regularization by truncated singular value decomposition (Bagshaw et al 2003). 


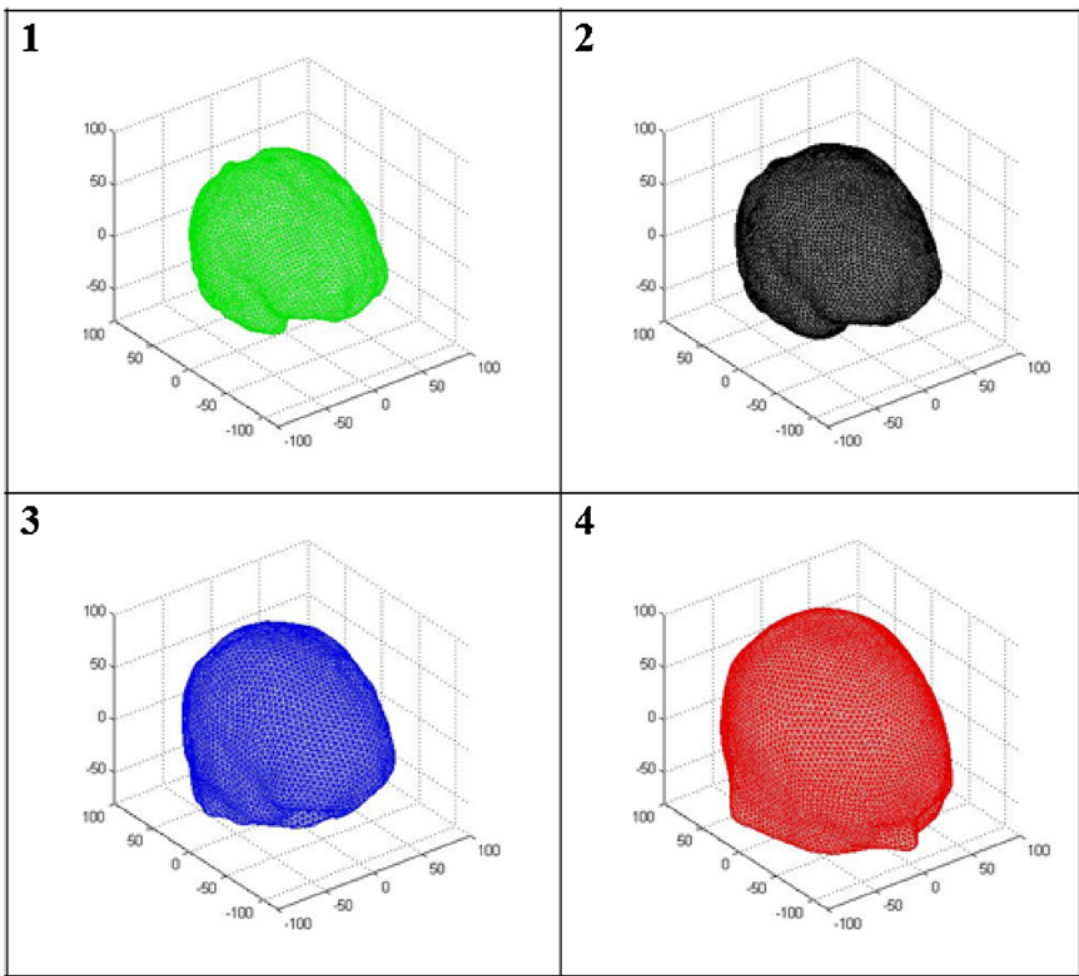

Figure 9. Example of four layer mesh-(1) Brain. (2) CSF. (3) Skull. (4) Scalp.

\section{Results}

\subsection{Resulting meshes}

Using a standard desktop, it was possible to generate accurate FE meshes from MRI and CT data in 30 and 60 min respectively. Seven randomly selected MRI scans (obtained from the BrainWeb database), and five CT scans were reconstructed (figures 9 and 10).

\subsection{Mesh quality}

For all meshes generated, the Jacobian and distortion metrics were within normal bounds (table 2). For the condition number and scaled Jacobian criteria, only a small percentage of elements were outside the acceptable limits. Note that the quality metrics of the meshes created from MRI were consistently better than those created from CT data.

\subsection{Simulations}

EIT images were reconstructed from simulated data using all the meshes generated with the proposed protocol and with a mesh that was manually segmented using I-DEAS from 2005 (Tizzard et al 2005). Noise was added up to $0.5 \%$ per channel. The resulting images were qualitatively similar for all the cases computed (figure 11). 


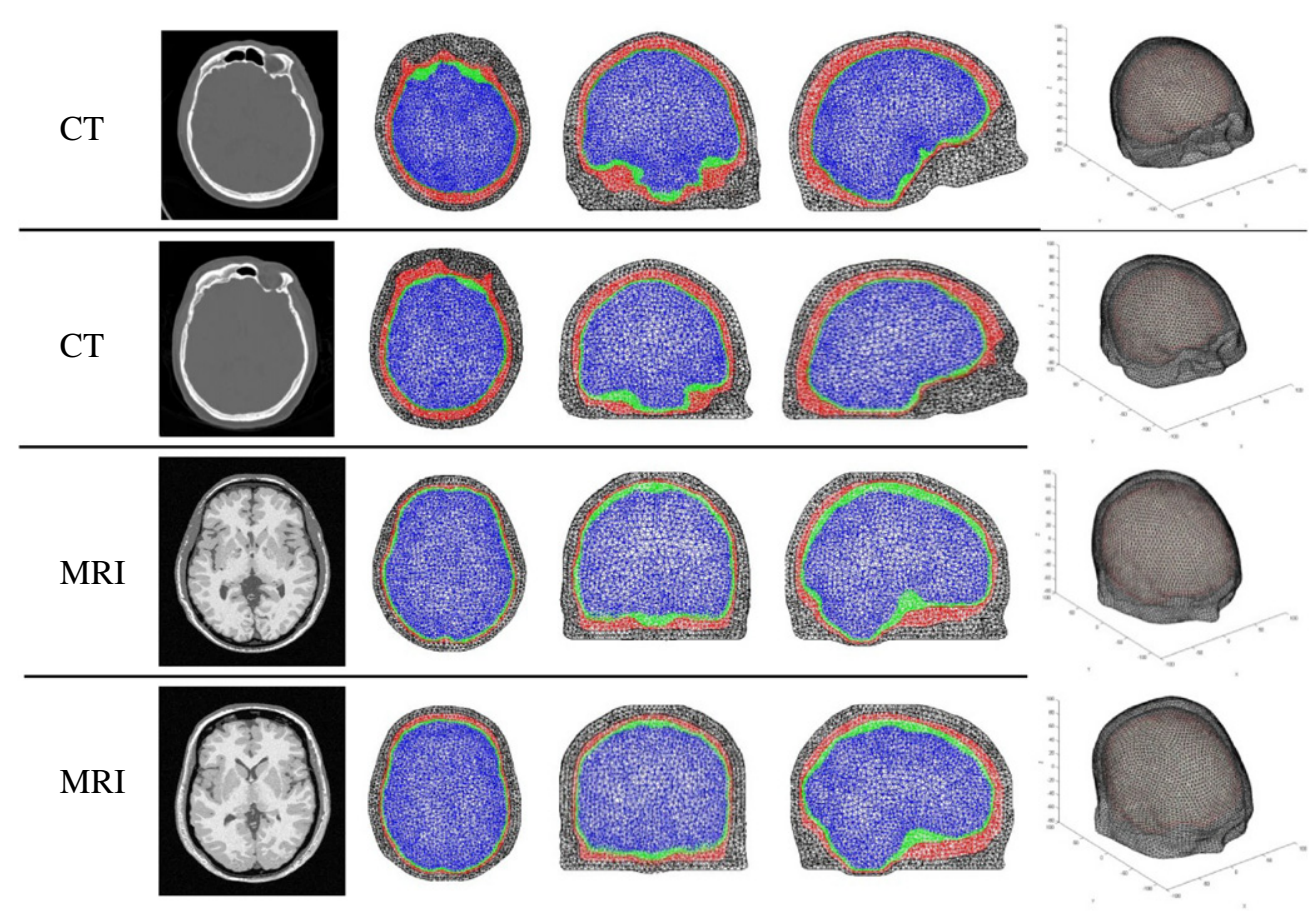

Figure 10. Four example four layer meshes — each row from left to right: original image data, three multi-planar views on the created mesh-scalp surface mesh.

Table 2. Number of elements that do not fulfil the quality requirements suggested by CUBIT. The limits of the measures are indicated in brackets.

\begin{tabular}{llllcl}
\hline Subject & $\begin{array}{l}\text { Jacobian } \\
\text { (not negative) }\end{array}$ & $\begin{array}{l}\text { Scaled } \\
\text { Jacobian }(0.2-1)\end{array}$ & $\begin{array}{l}\text { Distortion } \\
(0.6-1)\end{array}$ & $\begin{array}{l}\text { Condition } \\
\text { number (1-3) }\end{array}$ & $\begin{array}{l}\text { Number of } \\
\text { elements }\end{array}$ \\
\hline CT 1 & 0 & 109 & 0 & 61 & 331600 \\
CT 2 & 0 & 30 & 0 & 25 & 258798 \\
CT 3 & 0 & 303 & 0 & 237 & 313965 \\
CT 4 & 0 & 85 & 0 & 65 & 245826 \\
CT 5 & 0 & 262 & 0 & 89 & 222667 \\
CT 6 & 0 & 65 & 0 & 66 & 377512 \\
CT 7 & 0 & 331 & 0 & 315 & 276722 \\
MRI 1 & 0 & 6 & 0 & 21 & 261575 \\
MRI 2 & 0 & 5 & 0 & 19 & 300352 \\
MRI 3 & 0 & 3 & 0 & 8 & 248516 \\
MRI 4 & 0 & 11 & 0 & 26 & 312603 \\
MRI 5 & 0 & 11 & 0 & 16 & 319052 \\
\hline
\end{tabular}

\section{Discussion and conclusions}

\subsection{Summary of results}

The proposed method allows the rapid generation of accurate patient-specific meshes from MRI or CT scan data. Most of the mesh generation process was automated and used freely 

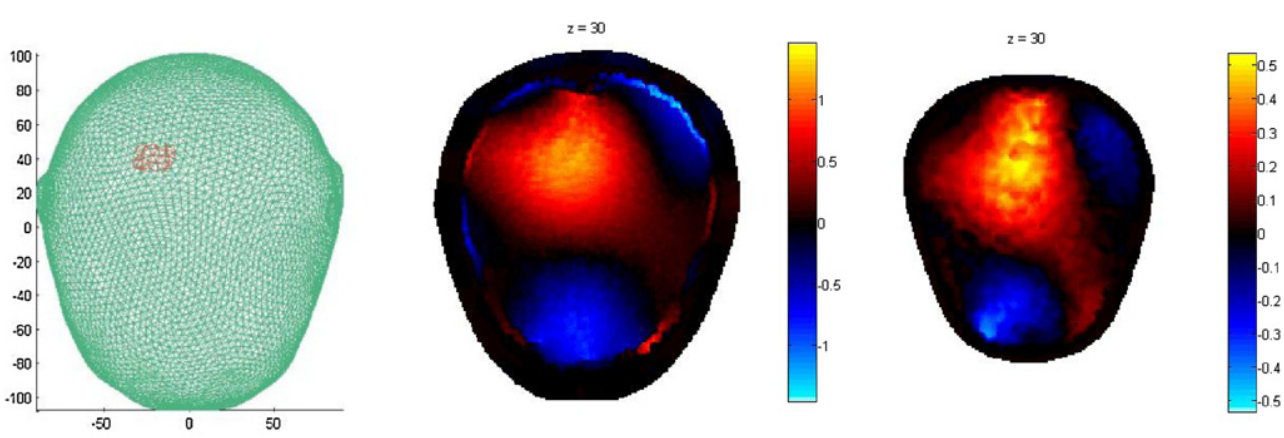

Figure 11. simulation results using up to $0.5 \%$ noise per channel; left: simulated perturbation area; middle—simulation using MRI mesh; right—simulation using validated I-DEAS mesh.

available user-friendly software. Other necessary custom scripts are provided as supplementary online data and are fully documented.

The generation of a subject-specific mesh from MRI and CT data took 30 and $60 \mathrm{~min}$ respectively. The resulting meshes exhibited high quality when tested with different criteria, and were found to be suitable for EIT brain imaging. Reconstructed images from simulated data appeared to be of satisfactory quality and closely resembled those from a previously employed manually produced mesh created using industry standard software.

\subsection{Technical considerations}

The file formats employed by BrainSuite and CUBIT were not compatible in detail, although similar in principle; this was easily solved by writing some Matlab conversion scripts. All scripts and custom written algorithms are available online and from the corresponding author upon request.

The programs are freely available on the internet, except for CUBIT and Matlab. Each employs a graphical user interface although CUBIT also requires some manual command entry. Although the use of several different programs might be confusing on first sight, we have now employed the procedure in our own laboratory and non-technical researchers can generally become familiar with the whole procedure in a few hours and are then able to produce patient specific meshes within about $1 \mathrm{~h}$.

\subsection{Recommendations for use}

The main purpose of this work is to describe a rapid and practicable protocol for generating accurate patient-specific meshes for EIT brain imaging. Investigation of recommended parameters for the different pieces of software has not been addressed exhaustively. However, visual inspection of the resulting meshes suggests that the standard settings (as suggested by the software) are reasonable. We found that a sampling interval of 6 during the isoparameterization resampling was a sensible trade-off between element size and computational time. Adaptive meshing using different sampling rates for different layers can be used to create smaller sized elements for thin layers.

Although this study focuses on the design of a protocol for accurate meshes for the human head, it could be eventually extended to other body regions. We hope that the proposed method 
would also be of interest for other imaging techniques such as optical tomography and EEG inverse source imaging.

\section{Acknowledgments}

The authors would like to thank Lior Horesh and Raya Schindmes who made valuable contributions at the outset of the development of this method.

\section{References}

Abascal J F, Arridge S R, Atkinson D, Horesh R, Fabrizi L, De Lucia M, Horesh L, Bayford R H and Holder D S 2008 Use of anisotropic modelling in electrical impedance tomography: description of method and preliminary assessment of utility in imaging brain function in the adult human head Neuroimage $43258-68$

Abascal J F, Arridge S R, Lionheart W R, Bayford R H and Holder D S 2007 Validation of a finite-element solution for electrical impedance tomography in an anisotropic medium Physiol. Meas. 28 S129-40

Adler A and Lionheart W R 2011 Minimizing EIT image artefacts from mesh variability in finite element models Physiol. Meas. 32 823-34

Arridge S R 1999 Optical tomography in medical imaging Inverse Problems 15 R41-93

Bagshaw A P, Liston A D, Bayford R H, Tizzard A, Gibson A P, Tidswell A T, Sparkes M K, Dehghani H, Binnie C D and Holder D S 2003 Electrical impedance tomography of human brain function using reconstruction algorithms based on the finite element method Neuroimage $20752-64$

Baillet S, Riera J J, Marin G, Mangin J F, Aubert J and Garnero L 2001 Evaluation of inverse methods and head models for EEG source localization using a human skull phantom Phys. Med. Biol. 46 77-96

Bonovas P M, Kyriacou G A and Sahalos J N 2001 A realistic three dimensional FEM of the human head Physiol. Meas. 22 65-76

Cardoso M J, Clarkson M J, Ridgway G R, Modat M, Fox N C and Ourselin S, The Alzheimer's Disease Neuroimaging Initiative 2011 LoAd: a locally adaptive cortical segmentation algorithm Neuroimage 56 1386-97

Drechsler F, Wolters C H, Dierkes T, SI H and Grasedyck L 2009 A full subtraction approach for finite element method based source analysis using constrained Delaunay tetrahedralisation Neuroimage 46 1055-65

Faugeras O et al 2004 Variational, geometric, and statistical methods for modeling brain anatomy and function Neuroimage 23 (Suppl. 1) S46-55

Functional Imaging Laboratory 2012 Statistical Parametric Mapping http://www.fil.ion.ucl.ac.uk/spm/

Gibson A P, Hebden J C and Arridge S R 2005 Recent advances in diffuse optical imaging Phys. Med. Biol. 50 R1-43

Gullmar D, Haueisen J and Reichenbach J R 2010 Influence of anisotropic electrical conductivity in white matter tissue on the EEG/MEG forward and inverse solution. A high-resolution whole head simulation study Neuroimage 51 145-63

Hallez H, Vanrumste B, Van Hese P, Delputte S and Lemahieu I 2008 Dipole estimation errors due to differences in modeling anisotropic conductivities in realistic head models for EEG source analysis Phys. Med. Biol. 53 1877-94

Holder D S 2005 Electrical Impedance Tomography: Methods, History and Applications (Bristol: CRC Press)

Itk-Snap 2011 ITK-Snap http://www.itksnap.org/

Kolehmainen V, Vauhkonen M, Karjalainen P A and Kaipio J P 1997 Assessment of errors in static electrical impedance tomography with adjacent and trigonometric current patterns Physiol. Meas. 18 289-303

Lew S, Wolters C H, Anwander A, Makeig S and Macleod R S 2009 Improved EEG source analysis using low-resolution conductivity estimation in a four-compartment finite element head model Hum. Brain Mapp. 30 2862-78

Liston A D, Bayford R H and Holder D S 2004 The effect of layers in imaging brain function using electrical impedance tomograghy Physiol. Meas. 25 143-58

Lorensen W E and Cline H E 1987 Marching cubes: a high resolution 3D surface construction algorithm Proc. of the 14th Annu. Conf. on Computer Graphics and Interactive Techniques ACM 163-69

Mathworks 2012 Matlab http://www.mathworks.com/products/matlab/

Mcconnell Brain Imaging Center 2006 BrainWeb Database http://www.bic.mni.mcgill.ca/brainweb/

MeshLab 2012 MeshLab http://meshlab.sourceforge.net/

Metherall P, Barber D C, Smallwood R H and Brown B H 1996 Three-dimensional electrical impedance tomography Nature 380 509-12

Modat M 2012 Nifty Reg http://www.cs.ucl.ac.uk/staff/m.modat/Marcs_Page/Software.html 
Neuroimaging Informatics Technology Initiative 2007 Nifty Format http://nifti.nimh.nih.gov/nifti-1/

Pietroni N, Tarini M and Cignoni P 2010 Almost isometric mesh parameterization through abstract domains IEEE Trans. Vis. Comput. Graphics 16 621-35

Ramon C, Schimpf P H and Haueisen J 2006 Influence of head models on EEG simulations and inverse source localizations Biomed. Eng. 510 PMID: 16466570

Rorden C 2012 MRIcro http://www.mricro.com/

Rullmann M, Anwander A, Dannhauer M, Warfield S K, Duffy F H and Wolters C H 2009 EEG source analysis of epileptiform activity using a $1 \mathrm{~mm}$ anisotropic hexahedra finite element head model Neuroimage 44 399-410

Sandia Corporation 2012 CUBIT Website http://cubit.sandia.gov/

Sandia National Laboratories 2012 http://www.sandia.gov/ [Accessed 11.03.2012]

Sazonov I and Nithiarasu P 2012 Semi-automatic surface and volume mesh generation for subject-specific biomedical geometries Int. J. Numer. Methods Biomed. Eng. 28 133-57

Shattuck D W 2012 BrainSuite Surface File Format http://users.loni.ucla.edu/ shattuck/brainsuite/file-formats/dfs/

Shattuck D W and Leahy R M 2002 BrainSuite: an automated cortical surface identification tool Med. Image Anal. 6 129-42

Shattuck D W, Sandor-LEAHY S R, Schaper K A, Rottenberg D A and Leahy R M 2001 Magnetic resonance image tissue classification using a partial volume model Neuroimage 13 856-76

Soni N K, Hartov A, Kogel C, Poplack S P and Paulsen K D 2004 Multi-frequency electrical impedance tomography of the breast: new clinical results Physiol. Meas. 25 301-14

Tidswell A T, Gibson A, Bayford R H and Holder D S 2001 Validation of a 3D reconstruction algorithm for EIT of human brain function in a realistic head-shaped tank Physiol. Meas. 22 177-85

Tizzard A, Horesh L, Yerworth R J, Holder D S and Bayford R H 2005 Generating accurate finite element meshes for the forward model of the human head in EIT Physiol. Meas. 26 S251-61

Towers C M, Mccann H, Wang M, Beatty P C, Pomfrett C J and Beck M S 2000 3D simulation of EIT for monitoring impedance variations within the human head Physiol. Meas. 21 119-24

UCL Custom 2012 Matlab Routines for Creation of FEM Meshes from MRI or CT http://www.ucl.ac.uk/silva/medphys/research/eit/resources/resourcepage

UCLA Laboratory of Neuro Imaging 2009 BrainSuite http://www.loni.ucla.edu/Software/BrainSuite

University College London 2012 http://cmic.cs.ucl.ac.uk/

Van Leemput K, Maes F, Vandermeulen D and Suetens P 1999 Automated model-based tissue classification of MR images of the brain IEEE Trans. Med. Imaging 18 897-908

Wideman G 2003 Analyze Format http://www.grahamwideman.com/gw/brain/analyze/formatdoc.htm

Wolters C H, Anwander A, Berti G and Hartmann U 2007 Geometry-adapted hexahedral meshes improve accuracy of finite-element-method-based EEG source analysis IEEE Trans. Biomed. Eng. 54 1446-53

Yerworth R J, Horesh L, Bayford R H, Tizzard A and Holder D S 2004 Robustness of linear and non-linear reconstructions algorithms for brain EITS. Non-linear-is it worth the effort? ICEBI'04: 12th Int. Conf. on Electrical Bio-Impedance joint with EIT-V Electrical Impedance Tomography (Gdansk, Poland) pp 499-502 (Available at http://eprints.mdx.ac.uk/2925/) 